\title{
Program Specification and Data Refinement in Type Theory
}

\author{
Zhaohui Luo \\ Department of Computer Science, University of Edinburgh \\ JCMB, KB, Mayfield Rd., Edinburgh EH9 3JZ, U.K.
}

\begin{abstract}
We develop a type-theoretic approach to program specification and data refinement and show that a type theory with a strong logical power and nice structural mechanisms provides an adequate formalism for modular development of programs and specifications. Specification of abstract data types is considered and a notion of abstract implementation between specifications is defined in the type theory and studied as a basis for correct and modular development of programs by stepwise refinement. The higher-order structural mechanisms in the type theory provide useful and flexible tools (specification operations and parameterized specifications) for modular design and structured specification. Refinement maps (programs and design decisions) and proofs of implementation correctness can be developed by means of the existing proof development systems based on type theories.
\end{abstract}

\section{Introduction}

Program specification and modular program development by stepwise refinement has been an interesting research area in computer science (see, for example, [Hoa72, LZ75, GHM76, GTW78, BG80, EFH83, MSV83, EM85, FGJM85, Wir86, Jon86, ST87, ST88a, WB89] among the enormous literature). Various formal abstraction mechanisms (e.g., algebraic specifications) have been studied to provide good methodology and tools which can be used to apply the useful principles of software development like separation of concerns and divide-and-conquer and to guarantee the correctness of programs with respect to their specifications.

Type theories (e.g., Martin-Löf's type theory [ML75, ML84], the Automath type theory [dB80], Nuprl's type theory [ $\left.\mathrm{C}^{+} 86\right]$, and Coquand-Huet's calculus of constructions [CH88]) were mainly developed for foundation and formalization of mathematics. Since the work by Martin-Löf, it has become known that type theories can also provide basic mechanisms for programming and program specification (c.f., [ML82, NPS90]). For instance, program derivation has been studied in various type theories [NPS90, BCM89, PM89]. However, although it is known that a type theory may be used as a programming and specification language, some important topics concerning modular design and structured specification (for example, abstract implementation and modular refinement) have not been paid enough attention in type-theoretic settings and the potential of type theory has not been well developed in this area.

In this paper, we develop a type-theoretic approach to program specification and data refinement and show that a type theory with nice structural mechanisms provides an adequate formalism for both modular 
design by data refinement and structured specification.

The type theory that we work with in this paper is the Extended Calculus of Constructions (ECC) [Lu089a, Luo90a]. As a formal system, ECC extends the calculus of constructions [CH88] with predicative type universes and $\Sigma$-types (strong sum); it may also be seen as an extension of Martin-Löf's type theory with universes [ML75] by an impredicative universe (higher-order logic). However, different from MartinLöf's type theory and the calculus of constructions, the incorporation of both an impredicative universe and predicative universes enhances a conceptual distinction between the notion of logical formulae (propositions) and that of sets (data types); this basic idea leads to a unifying theory of dependent types which provides not only strong logical power but also adequate abstraction mechanisms for pragmatic applications. One of the pragmatic motivations of the development of the theory ECC was to consider applications to program specification and abstract reasoning. The higher-order structural and logical mechanisms ( $\Sigma$-types and type universes, in particular) prove to be very useful for abstract reasoning [Luo89b] and program specification, the latter of which is discussed in this paper (also see [BM90]).

A specification in the type theory consists of (a pair of) a type, whose objects are the possible structures (program modules) which may realize the specification, and a predicate over the structure type, which specifies the properties that any realization should satisfy. In particular, the structure type of a specification of an abstract data type (say of stacks) can be defined as a $\Sigma$-type each of whose objects has as its components a type (of stacks) associated with an explicit congruence relation (between stacks) and certain operations (corresponding to the empty stack, push operation, etc.); the predicate over the structure type would specify the required properties including that the associated binary relation (between stacks) is a congruence. (Using an explicit congruence rather than a built-in equality is both adequate concerning about the semantics and important for stepwise abstract refinement. See section 3.) The semantics is straightforward and 'modeltheoretic' in the sense that a realization of a specification is simply a structure (an object of the structure type) which satisfies the required properties.

In order to discuss program development by stepwise refinement, we formalize a notion of abstract implementation between specifications which is similar to the notion of theory morphism for abstract reasoning (see [TL88, Luo89b]) and the notion of 'deliverables' by Burstall (see [BM90]). A specification $S P$ refines to (or is implemented by) another specification $S P^{\prime}$ through a refinement map $\rho$ (a function from the structure type of $S P^{\prime}$ to that of $S P$ ) if the images of $\rho$ over the realizations of $S P^{\prime}$ are realizations of $S P$. In such a case, the refinement map is an incomplete program which expresses the design decisions made in the refinement step. This implementation relation composes vertically (c.f., [BG80]) and hence satisfies the basic requirement for stepwise development of programs.

Based on the notion of abstract implementation, we further discuss methodological issues in software development and show that the higher-order structural mechanisms in the type theory nicely support modular design and structured specification. $\Sigma$-types support decomposition of specifications into independent specifications (which may possibly share some common parts). We also identify two general classes of specification operations, called constructors and selectors, which are monotone with respect to the implementation 
relation and can be used both in structured design by modular refinement and in structuring requirements specifications.

The higher-order facilities in the type theory naturally supports parameterized specifications. A notion of implementation between parameterized specifications is defined and it is shown to compose vertically. When a parameterized specification is monotone with respect to the implementation relation between specifications, the property of horizontal composition (c.f., [BG80]) holds and the design principle of divide-and-conquer can be applied as well.

The type-theoretic approach to specification and data refinement is simple and the higher-order mechanisms in the type theory provide powerful and useful supports in various aspects of modular development of programs and specifications. Note that the type theory provides one formal system in which specifications, programs and their implementation relationships can be uniformly formalized and discussed. Such an 'internalization' has an immediate benefit that refinement maps (programs and design decisions) as well as proofs of implementation correctness can be developed (interactively) in a proof development system like Lego [Pol89, LPT89], in which the type theory ECC is implemented. (In fact, all of the examples and propositions in this paper have been checked in the Lego system.) By this, we seem to have gained a good compromise between traditional model-theoretic approaches to specification semantics and the need to implement set theory in order to verify implementation correctness. We relate our type-theoretic approach to that of algebraic specifications, in particular by relating the notion of implementation to that of constructor implementation developed by Sannella and Tarlecki [ST88b].

In section 2, we briefly introduce the type theory used in this paper. Section 3 discusses specifications, specification of abstract data types, and the notion of abstract implementation. The issues about modular design and specification operations are dealt with in section 4 , and parameterized specifications and their implementations are discussed in section 5 . The work reported here is in progress; several further research topics are discussed in the conclusion.

\section{The Extended Calculus of Constructions}

As we have mentioned in the introduction, the type theory ECC [Luo89a, Luo90a] is a natural combination of Martin-Löf's type theory [ML75] and the calculus of constructions [CH88], based on the idea that there should be clear distinction between the notion of sets (data types) and that of logical formulae. The type system has good proof-theoretic properties (Church-Rosser, strong normalization, decidability and the others, see [Luo89a, Luo90a] for details) and a set-theoretic (realizability) model can be found in [Luo89b]. ECC is implemented in the proof development system Lego [Pol89, LPT89], which supports resolution-style interactive proof development. In this section, we give a brief and informal explanation of the type theory and introduce some notational conventions used in this paper.

We start by introducing the basic concepts and general rules. A context is a list of assumptions written in the form $x_{1}: A_{1}, \ldots, x_{n}: A_{n}(n \geq 0)$. There are two forms of judgements. The validity (well-formedness) of 
contexts is asserted by judgements of the form ' $\mathrm{T}$ valid' whose derivability is given by the following rules:

$$
\overline{() \text { valid }} \quad \frac{\Gamma \vdash A: T y p e_{i} \quad x \notin F V(\Gamma)}{\Gamma, x: A \text { valid }}
$$

where $\langle$ is the empty context and $F V(\Gamma)$ is the set of free variables in context $\Gamma$. A judgement of the form $\Gamma \vdash a: A$ (or simply $a: A$ when the context is clear or irrelevant ${ }^{1}$ ) means that object $a$ is of type $A$ (in context $\Gamma$ ). For example, having assumed $x: A$ in a valid context, we can derive that $x$ is an object of type $A$ in the context, as expressed by the following rule:

$$
\frac{\Gamma, x: A, \Gamma^{\prime} \text { valid }}{\Gamma, x: A, \Gamma^{\prime} \vdash x: A}
$$

A universe is a type which has (names of) types as its objects. Conversely, the types are the objects with universes as their types. The universes Prop and Type $(i \in \omega)$ are introduced by the following rules:

$$
\frac{\Gamma \text { valid }}{\Gamma \vdash \text { Prop }: \text { Type }} \quad \frac{\Gamma \text { valid }}{\Gamma \vdash T y p e_{i}: T y p e_{i+1}}
$$

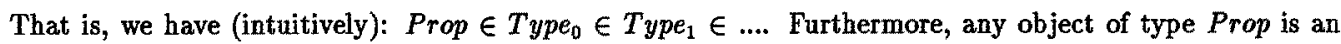
object of Type $_{0}$ and any object of type Type is an object of Type $e_{i+1}$; namely, Prop $\subseteq$ Type $e_{0} \subseteq T y p e_{1} \subseteq \ldots$ This type inclusion between universes, together with the intensional equality $\simeq$ (conversion, see below), generates a subtyping relation $\preceq$ between the types, ${ }^{2}$ which is formally expressed by the following rule:

$$
\frac{\Gamma \vdash a: A \quad \Gamma \vdash B: \text { Type }_{i}}{\Gamma \vdash a: B}(A \preceq B)
$$

As a special case, the above rule implies that two convertible types have the same objects. The universes in the type theory give a strong notion of type polymorphism and, together with the other type constructors, provide nice structural mechanisms.

Dependent product types (II-types) in the theory provide both dependent function spaces and the logical formulae of the embedded logic. The formation rules for II-types are

$$
\frac{\Gamma, x: A \vdash B: \text { Prop }}{\Gamma \vdash \Pi x: A . B: \text { Prop }} \quad \frac{\Gamma \vdash A: T y p e_{i} \quad \Gamma, x: A \vdash B: T y p e_{i}}{\Gamma \vdash \Pi x: A \cdot B: T y p e_{i}}
$$

They have the following introduction and elimination rules

$$
\frac{\Gamma, x: A \vdash b: B}{\Gamma \vdash \lambda x: A . b: \Pi \bar{\Pi} x: A . B} \quad \frac{\Gamma \vdash f: \Pi x: A . B \Gamma \vdash a: A}{\Gamma \vdash f(a):[a / x] B}
$$

and the $\beta$-conversion rule $(\lambda x: A . b)(a) \simeq[a / x] b$, where $[a / x]$ is the usual substitution operator.

Notation We may write $\Pi x: A . B$ as $A \rightarrow B$, when $B$ is not dependent on $x(x \notin F V(B))$. We also often

\footnotetext{
${ }^{1}$ In our discussion of specifications and program development in this paper, the reader may always assume that we are working in the empty context, unless we have made the context explicit.

${ }^{2}$ Since this is not important to understand the rest of this paper, we have intentionally omitted the technical definition of the subtyping relation. See [Luo89a, Luo90a] for details.
} 


$$
\begin{aligned}
& P_{1} \supset P_{2}=_{\text {df }} \quad P_{1} \rightarrow P_{2} \\
& \forall x: A \cdot P(x)={ }_{\mathrm{df}} \Pi \operatorname{II}: A \cdot P(x) \\
& \text { false }=_{\mathrm{df}} \quad \forall X: \text { Prop. } X \\
& P_{1} \& P_{2}={ }_{\mathrm{d} f} \quad \forall X: \text { Prop. }\left(P_{1} \supset P_{2} \supset X\right) \supset X \\
& P_{1} \vee P_{2}=\mathrm{df} \quad \forall X: \text { Prop. }\left(P_{1} \supset X\right) \supset\left(P_{2} \supset X\right) \supset X \\
& \neg P_{1} \quad=_{\mathrm{df}} \quad P_{1} \supset \text { false } \\
& \exists x: A . P(x)={ }_{\mathrm{d} f} \quad \forall X: P r o p .(\forall x: A .(P(x) \supset X)) \supset X \\
& a={ }_{A} b==_{\mathrm{d} f} \quad \forall P: A \rightarrow \text { Prop. } P(a) \supset P(b)
\end{aligned}
$$

Figure 1: Logical operators

write $f\left(a_{1}, \ldots, a_{n}\right)$ for $f\left(a_{1}\right) \ldots\left(a_{n}\right)$.

Note that the universes $T y p e_{i}$ are predicatively closed over products. For example, $\Pi x: T y p e_{i} \cdot x$ is of type

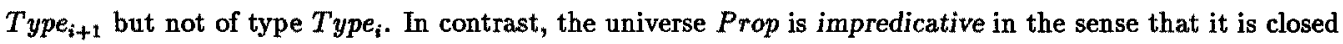
for arbitrary products. For example, Пx:Prop. $x$ is of type Prop. By the principle of propositions-as-types, this gives an embedded (intuitionistic) higher-order logic in the type theory, whose formulas are the objects of type Prop, called propositions. A proposition $P$ is provable if there is an object of type $P$. The logical constants and operators can be defined as shown in Figure 1, where $A$ is an arbitrary type, $P_{1}$ and $P_{2}$ are arbitrary propositions and $P$ is an arbitrary predicate over $A$ (a predicate over type $A$ is a propositional function of type $A \rightarrow$ Prop),$={ }_{A}$ as defined in Figure 1 is the so-called Leibniz's equality (between objects of type $A$ ). The Leibniz's equality reflects the basic computational equality (conversion) (that is, two closed terms are Leibniz's equal if and only if they are convertible [Luo90a]) and hence can be used to give adequate specifications of programs for concrete data types. The normalization theorem of the type system entails the consistency of the embedded logic [Luo90a]; furthermore, it is shown in [Luo90b] that this embedded logic is a conservative extension of the intuitionistic higher-order predicate logic (c.f., [Chu40]).

Remark Different from Martin-Löf's type theory [ML75, ML84], in ECC types and propositions are not identified and there is a distinguishable embedded logic. Every proposition is (lifted as) a type, but not vice versa. This gives a conceptual distinction between sets (data types), which reside in the predicative universes, and propositions (logical formulas), which reside in the impredicative universe. ${ }^{3}$ This distinction is important. Propositions should not be understood as arbitrary sets; to represent sets one should use predicative types. (See [Luo90b] for a discussion on this topic.)

\footnotetext{
${ }^{3}$ We can give a slightly different formulation of ECC by distinguishing the types with their names, which will make this point clearer. This is ont of the range of this paper.
} 
A strong sum type ( $\Sigma$-type) $\Sigma x: A . B(x)$ intuitively represents the set of pairs $(a, b)$, where $a$ is an object of type $A$ and $b$ an object of type $B(a)$. When $B$ is a predicate over $A$, it expresses the (intuitionistic) subset of the objects of type $A$ which satisfy the property $B$. The predicative universes are (predicatively) closed for strong sum:

$$
\frac{\Gamma \vdash A: T y p e_{i} \quad \Gamma, x: A \vdash B: T y p e_{i}}{\Gamma \vdash \Sigma x: A . B: T y p e_{i}}
$$

$\Sigma$-types have the following introduction and elimination rules

$$
\frac{\Gamma \vdash a: A \Gamma \vdash b:[a / x] B \Gamma, x: A \vdash B: T y p e_{i}}{\Gamma \vdash \operatorname{pair}_{\Sigma x: A . B}(a, b): \Sigma x: A . B} \quad \frac{\Gamma \vdash c: \Sigma x: A . B}{\Gamma \vdash \pi_{1}(c): A} \quad \frac{\Gamma \vdash c: \Sigma x: A . B}{\Gamma \vdash \pi_{2}(c):\left[\pi_{1}(c) / x\right] B}
$$

and the conversion rules $\pi_{i}\left(\operatorname{pair}_{A}\left(a_{1}, a_{2}\right)\right) \simeq a_{i}(i=1,2)$.

Notations We may write $\Sigma x: A . B$ as $A \times B$, when $B$ is not dependent on $x(x \notin F V(B))$. Although pairs in the type system is heavily typed (for decidability reasons), we often use $(a, b)$ rather than pair $_{A}(a, b)$ to denote pairs, when no confusion may occur. In this paper, we shall also use the following notational convention:

$$
\sum\left[x_{1}: A_{1}, x_{2}: A_{2}, \ldots, x_{n}: A_{n}\right]
$$

will denote the $\Sigma$-type

$$
\Sigma x_{1}: A_{1} \Sigma x_{2}: A_{2} \ldots \Sigma x_{n-1}: A_{n-1} \cdot A_{n}
$$

and, for any object $a$ of such a type, we use $x_{i}[a]$ as (the name of) the obvious projections over object $a$, e.g., $x_{2}[a]$ stands for $\pi_{1}\left(\pi_{2}(a)\right)$ and $x_{n}[a]$ for $\pi_{2}\left(\ldots \pi_{2}\left(\pi_{2}(a)\right) \ldots\right)$.

The predicative universes Type $_{i}$ can be viewed as set universes; the non-propositional types in predicative universes represent sets (or data types). (This is in contrast with the view of coding of data types [BB85] in an impredicative type system like Girard-Reynold's polymorphic $\lambda$-calculus [Gir72, Rey74] or the calculus of constructions [CH88].) These predicative universes are supposed to be open in the sense as Martin-Lof explains for his type theory [ML75, ML84]. For example, the type of natural numbers can be introduced by adding constants $\mathrm{N}:$ Type $_{0}, 0: \mathrm{N}$ and $s u c c: \mathrm{N} \rightarrow \mathrm{N}$, and a recursion operator rec $_{\mathrm{N}}$ with the elimination rule

$$
\frac{\Gamma \vdash C: \mathrm{N} \rightarrow T_{y p e_{j}} \Gamma \vdash c: C(0) \Gamma \vdash f: \Pi x: \mathrm{N} . C(x) \rightarrow C(\operatorname{succ}(x))}{\Gamma \vdash \operatorname{rec}_{\mathrm{N}}(c, f): \Pi x: \mathrm{N} . C(x)}
$$

and conversion rules $\operatorname{rec}_{\mathrm{N}}(c, f)(0) \simeq c$ and $\operatorname{rec}_{\mathrm{N}}(c, f)(\operatorname{succ}(x)) \simeq f\left(c, \operatorname{rec}_{\mathrm{N}}(c, f)(x)\right)$. Other data types like that of lists or type constructors may also be introduced in a similar way. An important topic which requires further research is how to introduce inductive types in a general way so that the good proof-theoretic properties still hold (see [CPM90, Ore90] where extensions of ECC by inductive types are discussed; also see [Luo90c] for a recent proposal using Martin-Löf's $W$-types). 
Concerning about the predicative universes, it is often tedious to always write the level subscripts. A technique has been developed [Hue87, HP89, Pol90] to ease the tension of worrying about universe levels so that, in practice, one can omit the universe levels to write Type instead of $T y p e_{i}$. This is nicely implemented in the proof development system Lego [Pol89, LPT89]. With such a facility, to assume $X: T y p e$ in a context is in some sense equivalent to assume that $X$ be an arbitrary type. One can also quantify over $T_{y p e}$ to talk about 'all types', bearing in mind that it is the machine who does the work to avoid universe circularity (by giving an error message when it occurs). In this paper, we shall adopt such a principle of 'typical ambiguity' to omit the universe subscripts.

\section{Specifications and Data Refinement in Type Theory}

It is well-known that in type theories one can specify programs (c.f., [ML82, NP83, NPS90]). For example, in Martin-Löf's type theory, a specification of sorting programs for lists of natural numbers may be defined as follows:

$$
\text { Sorting }=_{\mathrm{dr}} \Sigma f: \operatorname{List}(\mathbf{N}) \rightarrow \operatorname{List}(\mathbf{N}) . \forall l: \operatorname{List}(\mathbf{N}) . \operatorname{sorted}(l, f(l))
$$

The adequacy of such specifications (i.e., Sorting does specify the sorting programs) is in particular based on the fact that the propositional equality used in them reflects the computational equality, i.e., two closed programs are equal under the propositional equality if and only if they are computationally equal. (The need for this will become clear if one gives a full description of the predicate sorted in the above example). In ECC, Leibniz's equality (see section 2) can be used to describe the computational equality (conversion) based on an equality reflection result (see [Luo90a]). In Martin-Löf's type theory with weak intensional equality (see [NPS90]), the weak equality can be used to describe computation since it reflects the definitional equality. ${ }^{4}$

Note that specifications like the above directly specify programs for concrete data types (built-in in a type theory). Considering (large) program development by stepwise refinement, we are more interested in specifications of abstract data types with loose semantics. $\Sigma$-types provide a good mechanism to describe abstract structures and can be used to describe abstract data types. (See [NPS90] for an example of this, where the weak equality in Martin-Löf's type theory is used as the equality of the abstract data type.) However, carefully considering the (loose) semantics of abstract data types and the methodology of data refinement for program development, one will find out that specification of abstract data types has different requirements from those of programs for concrete data types discussed above. In particular, the equality over the abstract type should not be specified by a built-in equality like Leibniz's equality in our system (or the weak/strong equality in Martin-Löf's type theories); otherwise, we would not be able to refine a specification of an abstract data type to another in a stepwise way as expected and the best we could do would be to give a concrete realization of the specification.

\footnotetext{
${ }^{4}$ In Martin-Löf's type theory with strong extensional equality [ML84], the strong equality is essentially equivalent to the judgemental equality and the latter could be viewed as computational equality. Note that in the discussion here, we have taken a simple view without considering issues like observational equivalence.
} 
Therefore, instead of using a built-in equality as the equality for the carrier of an abstract data type, we associate the carrier type with an explicitly specified congruence relation to represent the intended equality. In what follows, after briefly explaining a general setting for specifications and semantics, we show by example how abstract data types can be specified, and then, we define a notion of abstract implementation between specifications and show how abstract refinement works.

\subsection{Specifications and abstract data types}

A specification in the type theory consists of (a pair of) a type, whose objects are the possible structures (program modules) which may realize the specification, and a predicate over the type, which specifies the properties that the realizations of the specification should satisfy.

Definition 3.1 (specifications) $A$ specification $S P$ consists of a type $\operatorname{Str}[S P]$ of $S P$-structures, called the structure type of $S P$, and a predicate $\mathbf{A x}[S P]$ over $\mathbf{S t r}[S P]$. The set of specifications is described by the following type:

$$
\mathrm{SPEC}=_{\mathrm{df}} \sum[\mathrm{Str}: \text { Type, Ax }: \mathrm{Str} \rightarrow \text { Prop }]
$$

For any type $S$, we also write $\operatorname{Spec}(S)$ for the type of specifications whose structure type is $S$.

Remark Note that a specification is not just a type, but a pair. The pragmatic significance of this is that we can separate computational contents (expressed by the structure type of a specification) from the axiomatic requirements for the programs (see also a remark in section 3.2). Such a separation is also the idea by Burstall on deliverables [BM90] and that for mathematical theories [Luo89b].

The semantics of specifications is determined by the type theory, as the following notions of realization and model give.

Definition 3.2 (realizations and models) Let $S P$ be a specification. $A$ realization (or concrete implementation) of $S P$ is an $S P$-structure $r$ (i.e, an object of type $\operatorname{Str}[S P]$ ) such that $\mathrm{Ax}[S P](r)$ is provable. $A$ model of $S P$ is an $S P$-structure together with the evidence that it satisfies $\mathrm{Ax}[S P]$; the set of $S P$-models are given by the following type:

$$
\operatorname{Mod}(S P)={ }_{\mathrm{df}} \Sigma s: \operatorname{Str}[S P] . \mathbf{A x}[S P](s)
$$

$S P$ is called realizable (or consistent) if there exists a realization, i.e., $\operatorname{Mod}(S P)$ is non-empty.

For example, a specification of the sorting programs for lists of natural numbers consists of the structure type $\operatorname{List}(\mathbf{N}) \rightarrow \operatorname{List}(\mathbf{N})$ and the predicate $\lambda f: \operatorname{List}(\mathbf{N}) \rightarrow \operatorname{List}(\mathrm{N}) . \forall l: \operatorname{List}(\mathbf{N})$. sorted $(l, f(l))$. A realization is just a sorting program. 
We now give an example to explain how abstract data types can be specified. We shall use the following definition in examples:

$$
\text { Setoid }=\text { df } \sum[D o m: T y p e, E q: D o m \rightarrow D o m \rightarrow \text { Prop }]
$$

A 'setoid' is a type together with a binary relation over the type. As we already mentioned above, an abstract type is always associated with a binary relation, which is a congruence relation as specified in the axiom part of specifications and is used to represent the intended equality over the abstract type.

Example 3.3 (Stack) A specification of stacks (of natural numbers) can be given as follows:

$$
\operatorname{Str}[\text { Stack }(\mathrm{N})]=\mathrm{df} \sum\left[\begin{array}{l}
\text { Stack }: \text { Setoid } \\
\text { empty }: \text { Dom }[\text { Stack }] \\
p u s h: \mathrm{N} \rightarrow \operatorname{Dom}[\text { Stack }] \rightarrow \text { Dom }[\text { Stack }] \\
p o p: D o m[\text { Stack }] \rightarrow \text { Dom[Stack }] \\
t o p: D o m[\text { Stack }] \rightarrow \mathrm{N}
\end{array}\right]
$$

and for any structure $S$ of type $\operatorname{Str}[\operatorname{Stack}(\mathbf{N})]$,

$$
\begin{aligned}
\operatorname{Ax}[\operatorname{Stack}(\mathrm{N})](S)=_{\mathrm{df}} & \operatorname{Cong}_{\mathrm{Stack}(\mathrm{N})}(E q) \\
& \& \quad E q(p o p(e m p t y), \text { empty }) \\
& \& \operatorname{top}(e m p t y)=_{\mathrm{N}} 0 \\
& \& \quad \forall n: \mathrm{N} \forall s: \operatorname{Dom}[\operatorname{Stack}] . \operatorname{Eq}(\operatorname{pop}(p u s h(n, s)), s) \\
& \& \quad \forall n: \mathrm{N} \forall s: \operatorname{Dom}[\operatorname{Stack}] \cdot \operatorname{top}(\operatorname{push}(n, s))={ }_{\mathrm{N}} n
\end{aligned}
$$

where Stack abbreviates $S t a c k[S], E q$ abbreviates $E q[S t a c k[S]]$, and similar for the others; $\operatorname{Cong}_{\text {stack(N) }}(E q)$ stands for the following proposition expressing that the binary relation between stacks is a congruence:

$$
\begin{array}{rl}
\operatorname{Cong}_{\text {Stack }(\mathrm{N})}(E q)==_{\mathrm{df}} & E q u i v(E q) \& \\
& \forall s, s^{\prime}: \operatorname{Dom}[S t a c k] . \quad E q\left(s, s^{\prime}\right) \supset \\
& \operatorname{top}(s)=_{\mathrm{N}} \operatorname{top}\left(s^{\prime}\right) \& E q\left(p o p(s), p o p\left(s^{\prime}\right)\right) \& \\
& \forall m, n: \mathrm{N} . m={ }_{\mathrm{N}} n \supset E q\left(p u s h(m, s), \operatorname{push}\left(n, s^{\prime}\right)\right)
\end{array}
$$

Note that, in the above example, we only require that the equality $E q$ between stacks be a congruence. This enables further refinement of the specification by another (for example, arrays of natural numbers, see below). If we had instead used Leibniz's equality, such an abstract refinement would not be possible.

\subsection{Data refinement and implementation}

A notion of (abstract) implementation is the most important if we want to develop programs in a stepwise way by refinement between specifications. That is, we want to know what we mean by saying that a specification 
refines to (or is implemented by) another specification. Various notions have been proposed in the literature, starting from the early notion of abstraction function and representation function (see [Hoa72, GTW78]) to the more recent considerations (see, for example, [ST88b, WB89]). We formalize in the type theory a simple notion of refinement (implementation). This notion comes from the consideration of theory morphisms in abstract reasoning [TL88, Luo89b] and is similar to the notion of deliverables [BM90].

Definition 3.4 (refinement map and implementation) Let $S P$ and $S P^{\prime}$ be specifications. $A$ refinement map from $S P^{\prime}$ to $S P$ is a functional operation $\rho$ from $\operatorname{Str}\left[S P^{\prime}\right]$ to $\operatorname{Str}[S P]$,

$$
\rho: \operatorname{Str}\left[S P^{\prime}\right] \rightarrow \operatorname{Str}[S P]
$$

such that the following satisfaction condition is provable:

$$
\operatorname{Sat}(\rho)=_{\mathrm{df}} \forall s^{\prime}: \operatorname{Str}\left[S P^{\prime}\right] \cdot \mathbf{A x}\left[S P^{\prime}\right]\left(s^{\prime}\right) \supset \mathbf{A x}[S P]\left(\rho\left(s^{\prime}\right)\right)
$$

If $\rho$ is a refinement map from $S P^{\prime}$ to $S P$, we say that $S P$ refines to (or is implemented by) $S P^{\prime}$ through $\rho$, notation $S P \Longrightarrow_{p} S P^{\prime}$.

Notation If $\operatorname{Str}[S P]=\operatorname{Str}\left[S P^{\prime}\right]$ and id $: \operatorname{Str}\left[S P^{\prime}\right] \rightarrow \operatorname{Str}[S P]$ is the identity function, we may write $S P \Longrightarrow S P^{\prime}$ to abbreviate $S P \Longrightarrow_{i d} S P^{\prime}$.

Remark Note that refinement maps are incomplete programs and incorporate various design decisions during the process of refinement implementation. One might consider a seemingly simpler and more general notion by defining a refinement map simply as a function of type $\operatorname{Mod}\left(S P^{\prime}\right) \rightarrow \operatorname{Mod}(S P)$ with certain proofirrelevance condition. However, such a notion would not distinguish the computational part of an abstract implementation from its non-computational contents (correctness proofs) as ours does. Such a separation is very important when considering development of programs, since refinement maps (programs) should not contain unnecessary components concerning about (proofs of) implementation correctness. We also believe, at least in practice, that (in an intuitionistic setting) every function comes from an extensional operation and our notion of refinement is general enough and adequate. (See [Bee82] for a relevant discussion on the relationship between functions and extensional operations.)

Informally, a refinement map from $S P^{\prime}$ to $S P$ determines the following subset of realizations of the original specification $S P$ :

$$
\left\{\rho\left(m^{\prime}\right) \mid \exists p .\left(m^{\prime}, p\right) \in \operatorname{Mod}\left(S P^{\prime}\right)\right\}
$$

i.e., the images of the refinement map over the realizations of $S P^{\prime}$. The stepwise development of programs from a specification $S P_{0}$ would be a sequence of refinement implementation steps:

$$
S P_{0} \Longrightarrow_{\rho_{1}} S P_{1} \Longrightarrow_{\rho_{2}} \ldots \Longrightarrow_{\rho_{n}} S P_{n}
$$


Any realization $r_{n}$ of $S P_{n}$ gives a realization $r_{0}$ of $S P_{0}$ as,

$$
r_{0}=\rho_{1}\left(\ldots\left(\rho_{n}\left(r_{n}\right)\right)\right)
$$

and, the composition of the correctness proofs for each step will give the proof that $r_{0}$ is a realization of $S P_{0}$. This justifies the fact that the implementation relation composes vertically (c.f., [BG80]).

Proposition 3.5 (vertical composition) If $S P \Longrightarrow_{\rho} S P^{\prime}$ and $S P^{\prime} \Longrightarrow_{\rho^{\prime}} S P^{\prime \prime}$, then $S P \Longrightarrow \Longrightarrow_{\rho \circ \rho^{\prime}} S P^{\prime \prime}$, where $\rho \circ \rho^{\prime}=\lambda s^{\prime \prime}: \operatorname{Str}\left[S P^{\prime \prime}\right] . \rho\left(\rho^{\prime}\left(s^{\prime \prime}\right)\right)$ is the functional composition of $\rho$ and $\rho^{\prime}$.

Remark As a special case of the above proposition, $S P \Longrightarrow S P^{\prime}$ and $S P^{\prime} \Longrightarrow S P^{\prime \prime}$ imply $S P \Longrightarrow S P^{\prime \prime}$.

Let us now see a traditional example of refinement - implementing stacks by array-pointer pairs.

Example 3.6 We consider a specification Array(N) for arrays (of natural numbers), define a refinement map $\rho$ from $\operatorname{Array}(\mathbf{N})$ to the specification Stack(N) defined in Example 3.3 such that Stack(N) is implemented by $\operatorname{Array}(\mathbf{N})$ through $\rho$. The specification of $\operatorname{Array}(\mathbf{N})$ is given by

$$
\operatorname{Str}[\text { Array }(\mathbf{N})]=\mathrm{df} \sum\left[\begin{array}{l}
\text { Array }: \text { Setoid } \\
\text { newarray }: \text { Dom }[\text { Array }] \\
\text { assign }: \mathrm{N} \rightarrow \text { Dom }[\text { Array }] \rightarrow \mathrm{N} \rightarrow \text { Dom }[\text { Array }] \\
\text { access }: \text { Dom }[\text { Array }] \rightarrow \mathrm{N} \rightarrow \mathrm{N}
\end{array}\right]
$$

and, for any structure $A$ of type $\operatorname{Str}[\operatorname{Array}(\mathbf{N})]$,

$$
\begin{aligned}
& \operatorname{Ax}[\operatorname{Array}(\mathbf{N})](A)=_{\mathrm{df}} \quad \operatorname{Cong}_{\operatorname{Array}(\mathbb{N})}(E q) \\
& \text { \& } \forall i: N . \operatorname{access}(\text { newarray, } i)={ }_{\mathrm{N}} 0 \\
& \& \quad \forall n, i, j: \mathbf{N} \forall a: D o m[\text { Array]. } \\
& (i=\mathrm{N} j \supset \operatorname{access}(\operatorname{assign}(n, a, i), j)=\mathrm{N} n) \\
& \&\left(i \neq \neq_{\mathrm{N}} j \supset \operatorname{access}(\operatorname{assign}(n, a, i), j)=_{\mathrm{N}} \operatorname{access}(a, j)\right)
\end{aligned}
$$

where Cong $\operatorname{Array}_{(\mathrm{N})}(E q)$ is the proposition expressing that the relation $E q$ between arrays is a congruence, similarly defined as Congstack $(\mathrm{N})(E q)$ in Example 3.3 .

The refinement map $\rho$, given any Array(N)-structure $A$, generates the following $\operatorname{Stack}(\mathbf{N})$-structure:

$$
\begin{aligned}
& \operatorname{Dom}[\operatorname{Stack}[\rho(A)]]=\text { df } \sum[\operatorname{arr}: \operatorname{Dam}[\operatorname{Array}[A]], \operatorname{ptr}: \mathbf{N}] \\
& E q[\operatorname{Stack}[\rho(A)]] \quad=_{\mathrm{df}} \lambda s, s^{\prime}: \operatorname{Dom}[\operatorname{Stack}[\rho(A)]] \text {. } \\
& \operatorname{ptr}[s]={ }_{\mathrm{N}} p t r\left[s^{\prime}\right] \& \\
& \forall i: N . i<\operatorname{ptr}[s] \supset \operatorname{access}(\operatorname{arr}[s], i)={ }_{\mathrm{N}} \operatorname{access}\left(\operatorname{arr}\left[s^{\prime}\right], i\right) \\
& \operatorname{empty}[\rho(A)] \quad={ }_{\mathrm{d} f}(\text { newarray }[A], 0) \\
& \operatorname{push}[\rho(A)] \quad={ }_{\mathrm{df}} \quad \lambda n: \mathrm{N} \lambda s: \operatorname{Dom}[\operatorname{Stack}[\rho(A)]] \cdot(\operatorname{assign}(n, \operatorname{arr}[s], p t r[s]), p t r[s]+1) \\
& \operatorname{pop}[\rho(A)] \quad=\mathrm{df} \quad \lambda s: \operatorname{Dom}[\operatorname{Stack}[\rho(A)]] \cdot(\operatorname{arr}[s], p \operatorname{tr}[s]-1) \\
& \operatorname{top}[\rho(A)] \quad=\mathrm{df} \quad \lambda s: \operatorname{Dom}[\operatorname{Stack}[\rho(A)]] \cdot \operatorname{access}(\operatorname{arr}[s], p \operatorname{tr}[s]-1)
\end{aligned}
$$


Note that the 'equality representation' (or 'representation invariants') in the traditional approaches to proofs of abstract implementation is directly reflected in the refinement map (its $E q[S t a c k[\rho(A)]]$ part). This is possible because we have associated the explicit congruences in the specifications rather than using a fixed equality for the abstract type.

$\rho$ defined above is indeed a refinement map from $\operatorname{Array}(\mathbf{N})$ to $\operatorname{Stack}(\mathbf{N})$.

Fact 3.7 Stack(N) $\Longrightarrow_{\rho} \operatorname{Array}(N)$.

An obviously important issue in a refinement development of programs is that the correctness of the (abstract) implementations must be verified. In other words, the satisfaction condition must be proved. Proof development systems based on type theories like Lego [Pol89, LPT89] can be used to verify the correctness of implementation. The above fact of implementation correctness has been formally checked in Lego; in fact, we have used Lego to develop the refinement map interactively on the machine.

\section{Modular Design and Structured Specification}

Modular design and structured specification have been generally accepted as two related useful methodologies for software development. There are two issues here. First, given a requirements specification to be implemented, programmers use principles like divide-and-conquer and stepwise refinement to decompose and refine the specification until they reach desirable low-level specifications which can be concretely implemented by, say efficient enough, software modules. This is the process of modular design, which may involve many intermediate design specifications, probably proposed by some chief programmers and further implemented separately by others. Secondly, to get a good requirements specification of a large software system, people must structure the specification in a modular way so that it is understandable and may suggest some possible design decisions.

Having given a notion of implementation in the last section, we discuss in this section modular design and structured specification in our type-theoretic approach (parameterized specifications are discussed in section 5 ). In particular, we consider various specification operations ${ }^{5}$ which can be used either in structured design by refinement or in structuring requirements specifications. An important property of such specification operations is the monotonicity with respect to the implementation relation (see below), which will ensure independent further refinements of the argument specifications (the so-called horizontal composition property [BG80]).

\subsection{Decomposition and sharing}

Using the principle of divide-and-conquer in a design process, developers often decompose a specification into several independent ones (with clear interfaces) so that they can be implemented separately and their

\footnotetext{
${ }^{5}$ A specification operation is a fonction which takes specifications (and possibly some other kinds of objects) as arguments and returns a specification as the result of application. Specification operations can also be seen as parameterized specifications. See section 5 .
} 
realizations can be put together to get a realization of the original specification. Considering this, one might think that the notion of implementation given in the last section is over-simplified since at first appearance it seemed to cover only the situations where a single line of refinement is pursued. In fact, this is not the case. Using the notion of implementation and $\Sigma$-types in our type theory, one can do specification decomposition by considering the following specification operation. (This also gives us a simple example to explain why the monotonicity of specification operations offers the independency for further refinements.)

Definition 4.1 Let $S P$ and $S P^{\prime}$ be specifications. Then, specification $S P \otimes S P^{\prime}$ is defined as follows:

$$
\operatorname{Str}\left[S P \otimes S P^{\prime}\right]=_{\mathrm{df}} \operatorname{Str}[S P] \times \operatorname{Str}\left[S P^{\prime}\right]
$$

and, for any $s$ of type $\mathbf{S t r}\left[S P \otimes S P^{\prime}\right]$,

$$
\mathbf{A x}\left[S P \otimes S P^{\prime}\right](s)=_{\mathrm{df}} \mathbf{A x}[S P]\left(\pi_{1}(s)\right) \& \operatorname{Ax}\left[S P^{\prime}\right]\left(\pi_{2}(s)\right)
$$

The (infix) specification operation $\otimes$ is of type SPEC $\rightarrow$ SPEC $\rightarrow$ SPEC.

The idea to use the above specification operation to decompose a specification $S P$ into several (say two) independent specifications $\left(S P_{1}\right.$ and $\left.S P_{2}\right)$ by certain design strategy is to consider a refinement step of the following form:

$$
S P \Longrightarrow S P_{1} \otimes S P_{2}
$$

where the refinement map $\rho: \operatorname{Str}\left[S P_{1} \otimes S P_{2}\right] \rightarrow \operatorname{Str}[S P]$ is the incomplete program expressing the design strategy at this decomposition step. The intention here is to implement the specifications $S P_{1}$ and $S P_{2}$ independently by further refinements. The soundness for such independent further refinements is guaranteed by the monotonicity of $\otimes$ with respect to the implementation relation.

Proposition 4.2 (monotonicity of $\otimes$ ) If $S P_{i} \Rightarrow_{\rho_{i}} S P_{i}^{\prime}(i=1,2)$, then $S P_{1} \otimes S P_{2} \Longrightarrow_{\rho_{1} \otimes \rho_{2}} S P_{1}^{\prime} \otimes S P_{2}^{\prime}$, where $\rho_{1} \otimes \rho_{2}=\lambda s^{\prime}: \operatorname{Str}\left[S P_{1}^{\prime} \otimes S P_{2}^{\prime}\right] \cdot\left(\rho_{1}\left(\pi_{1}\left(s^{\prime}\right)\right), \rho_{2}\left(\pi_{2}\left(s^{\prime}\right)\right)\right)$.

By the monotonicity of $\otimes$ and the vertical composition property of the implementation relation (Proposition 3.5), we have that $S P \Longrightarrow_{\rho} S P_{1} \otimes S P_{2}$ and $S P_{i} \Longrightarrow_{\rho_{1}} S P_{i}^{\prime}(i=1,2)$ imply $S P \Longrightarrow_{\rho \circ\left(\rho_{1} \otimes \rho_{2}\right)} S P_{1}^{\prime} \otimes S P_{2}^{\prime}$ and, for any realizations $r_{i}^{\prime}$ of $S P_{i}^{\prime}(i=1,2), \rho \circ\left(\rho_{1} \otimes \rho_{2}\right)\left(r_{1}^{\prime}, r_{2}^{\prime}\right)$ is a realization of $S P$.

In our discussion so far, we have only considered decomposition of a specification into several completely independent specifications. In practice, it is often the case that the sub-specifications are not completely independent but share some common structure. ${ }^{6}$ For example, one may decompose a specification of a parser into several specifications including $A b s S y n$ for an abstract syntax tree generator and $S y m T a b$ for management of the symbol table; these later two specifications both use symbols and symbol management functions which are specified by another specification Symbol. Note that a parser can only work correctly when $A b s S y n$ and SymTab use the same realization of Symbol. Such a structure sharing can be dealt with by using $\Sigma$-types by considering the following specification operation $\sum$, which has $\otimes$ above as a special case.

\footnotetext{
${ }^{8}$ Much attention has been paid to such structure sharing in the design of both programming languages (e.g., Standard ML [Mac81, MTH90] and Pebble [BL84]) and specification languages (e.g., Clear [BG80] and Extended ML [ST87]).
} 
Definition 4.3 Let $S P$ be a specification and $P: \operatorname{Str}[S P] \rightarrow \operatorname{SPEC}$. Then, $\Sigma(S P, P)$ is the specification defined as follows:

$$
\operatorname{Str}[\Sigma(S P, P)]=_{\mathrm{df}} \Sigma s: \operatorname{Str}[S P] . \operatorname{Str}[P(s)]
$$

and, for any $s^{\prime}$ of type $\operatorname{Str}[\Omega(S P, P)]$,

$$
\operatorname{Ax}\left[\sum(S P, P)\right]\left(s^{\prime}\right)=_{\mathrm{df}} \operatorname{Ax}[S P]\left(\pi_{1}\left(s^{\prime}\right)\right) \& \operatorname{Ax}\left[P\left(\pi_{1}\left(s^{\prime}\right)\right)\right]\left(\pi_{2}\left(s^{\prime}\right)\right)
$$

$\sum$ is of type IISP:SPECחP:Str[SP] $\rightarrow$ SPEC. SPEC.

Proposition 4.4 (monotonicity of $£$ ) Let $S P$ and $S P^{\prime}$ be specifications, $P: \operatorname{Str}[S P] \rightarrow \mathrm{SPEC}$ and $P^{\prime}: \operatorname{Str}\left[S P^{\prime}\right] \rightarrow$ SPEC. If

1. $S P \Longrightarrow_{\rho} S P^{\prime}$, and

2. $\delta$ is a function of type $\Pi s^{\prime}: \operatorname{Str}\left[S P^{\prime}\right] . \operatorname{Str}\left[P^{\prime}\left(s^{\prime}\right)\right] \rightarrow \operatorname{Str}\left[P\left(\rho\left(s^{\prime}\right)\right)\right]$ such that $\forall s^{\prime}: \operatorname{Str}\left[S P^{\prime}\right] . P\left(\rho\left(s^{\prime}\right)\right) \Longrightarrow_{\delta\left(s^{\prime}\right)}$ $P^{\prime}\left(s^{\prime}\right)$ is provable,

then,

$$
\sum(S P, P) \Longrightarrow \sum(0, \delta) \sum\left(S P^{\prime}, P^{\prime}\right)
$$

where $\Sigma(\rho, \delta)=\lambda s^{\prime}: \operatorname{Str}\left[\Sigma\left(S P^{\prime}, P^{\prime}\right)\right] \cdot\left(\rho\left(\pi_{1}\left(s^{\prime}\right)\right), \delta\left(\pi_{1}\left(s^{\prime}\right), \pi_{2}\left(s^{\prime}\right)\right)\right)$.

Suppose $P$ is of the form $\lambda s_{0}: \operatorname{Str}\left[S P_{0}\right] . P_{1}\left(s_{0}\right) \otimes P_{2}\left(s_{0}\right)$. Then, a refinement step

$$
S P \Longrightarrow \varnothing\left(S P_{0}, P\right)
$$

decomposes $S P$ into three specifications $S P_{0}, P_{1}\left(s_{0}\right)$ and $P_{2}\left(s_{0}\right)$; the latter two share a common structure specified by $S P_{0}$. The above monotonicity result suggests one to decompose $S P$ into $S P_{0}$ and (parameterized specification) $P$ which are independent of each other (see section 5). Another way to look at the further refinement of $\Phi\left(S P_{0}, P\right)$ is to consider $S P_{0}$ and the following specification $S P^{\prime}$;

$$
\begin{gathered}
\operatorname{Str}\left[S P^{\prime}\right]==_{\mathrm{df}} \Pi s_{0}: \operatorname{Str}\left[S P_{0}\right] . \operatorname{Str}\left[P\left(s_{0}\right)\right] \\
\operatorname{Ax}\left[S P^{\prime}\right\}(f)=_{\mathrm{df}} \forall m: \operatorname{Mod}\left(S P_{0}\right) \cdot \operatorname{Ax}\left[P\left(f\left(\pi_{1}(m)\right)\right)\right]\left(f\left(\pi_{1}(m)\right)\right)
\end{gathered}
$$

In other words, we proceed to implement $S P_{1}\left(s_{0}\right)$ and $S P_{1}\left(s_{0}\right)$ independently, assuming that $s_{0}$ is an arbitrary realization of $S P_{0}$. Note that $S P_{0}$ and $S P^{\prime}$ are independent of each other and they have a clear interface specified by $\Pi$. To get a realization of $\varrho\left(S P_{0}, P\right)$, we simply put together any realization $r_{0}$ of $S P_{0}$ and the result of applying any realization of $S P^{\prime}$ to $r_{0}$.

Warning: Not all such decompositions can lead to solutions; in other words, one may go into a blind alley

\footnotetext{
${ }^{7} P$ and $P^{\prime}$ are parameterized specifications and, when $S P$ and $S P^{\prime}$ have the same structure type and $\rho$ is the identity function, the condition for $\delta$ here is to say that the parameterized specification $P$ refines to $P^{\prime}$ through $\delta$. See Definition 5.2 in section 5 .
} 
- some of the sub-specifications are not realizable. Here is a trivial example: Decomposing a specification whose structure type is $\Sigma x:$ Prop. $x$ into two specifications with Prop and $\Pi x:$ Prop. $x$ as structure types, respectively, produces an inconsistent specification (the second one) since $\Pi x:$ Prop. $x$ has no object in any consistent context in the type theory. In any stage of refinement development, the programmer must be careful about such a consistency argument. If necessary, one may verify that certain intermediate design specifications are realizable. For the above situation concerning about sharing, if independent decomposition is not feasible, we have to first refine $S P_{0}$ to $S P_{0}^{\prime}$ (with the same structure type) so that such a decomposition for $E\left(S P_{0}^{t}, P\right)$ is possible, or we simply find an (intended) realization $r_{0}$ of $S P_{0}$ and then implement $P\left(r_{0}\right)$.

\subsection{Constructors and selectors}

Since the work by Burstall and Goguen on specification language Clear [BG80], it has been generally accepted that specification operations play important roles both in modular design by refinement and in structuring specifications. For example, we can define the following simple specification operations which may often be used in structuring specifications:

- Join ${ }_{S}$ : it 'puts together' the axiomatic parts of two specifications over the same structure type $S$ : if $\operatorname{Str}[S P]=\operatorname{Str}\left[S P^{\prime}\right]=S$, then

$$
\begin{aligned}
& \operatorname{Str}\left[\operatorname{Join}_{S}\left(S P, S P^{\prime}\right)\right]==_{\mathrm{df}} S \\
& \mathbf{A x}\left[\operatorname{Join}_{s}\left(S P, S P^{\prime}\right)\right](s)={ }_{\mathrm{d} f} \quad \mathbf{A x}[S P](s) \& \mathbf{A x}\left[S P^{\prime}\right](s)
\end{aligned}
$$

Join is of type

$$
\text { IIS:Type. } \operatorname{Spec}(S) \rightarrow \operatorname{Spec}(S) \rightarrow \operatorname{Spec}(S)
$$

Similarly, one may define $\operatorname{Meet}_{S}$ with $\operatorname{Ax}\left[\operatorname{Meet}_{S}\left(S P, S P^{\prime}\right)\right](s)={ }_{\mathrm{d} f} \operatorname{Ax}[S P](s) \vee \operatorname{Ax}\left[S P^{\prime}\right](s)$, and other possibly useful operators by means of logical operators.

- Extend: it extends a specification by some extra structure-components and/or some axioms. Given a specification $S P$, an extension Ext_Str of $\operatorname{Str}[S P]$, which is a function of $\operatorname{type} \operatorname{Str}[S P] \rightarrow T y p e$, and some axioms (a predicate) Ext_Ax over the extended structure type (i.e., $\Sigma s: \operatorname{Str}[S P] . E x t_{-} S t r(s)$ )

$$
\begin{aligned}
\operatorname{Str}\left[\operatorname{Extend}\left(S P, E x t_{-} S t r, E x t_{-} A x\right)\right] & =_{\mathrm{d} f} \quad \Sigma s: \operatorname{Str}[S P] . \operatorname{Ext} S \operatorname{Str}(s) \\
\operatorname{Ax}\left[\operatorname{Extend}\left(S P, E x t_{-} S t r, E x t_{-} A x\right)\right]\left(s^{\prime}\right) & { }_{\mathrm{d} f} \operatorname{Ax}[S P]\left(\pi_{1}\left(s^{\prime}\right)\right) \& E x t_{-} A x\left(s^{\prime}\right)
\end{aligned}
$$

Extend is of type

$$
\text { ПISP:SPECח } f: \operatorname{Str}[S P] \rightarrow T y p e \Pi g:(\Sigma s: \operatorname{Str}[S P] . f(s)) \rightarrow \text { Prop. SPEC }
$$

There are various specification operations which can be defined. Instead of studying them one by one (e.g., considering whether they are monotone), we define two general classes of specification operations called 
constructors $^{8}$ and selectors, which are determined by functions between structure types.

Definition 4.5 (constructors and selectors) Let $S$ and $S^{\prime}$ be types and $\rho: S^{\prime} \rightarrow S$. The specification operations Con $\mathrm{C}_{\rho}$ and $\mathrm{Sel}_{\rho}$ are defined as follows:

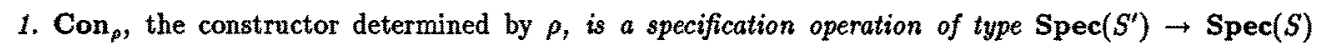
defined as: for any $S P^{\prime}$ with $\operatorname{Str}\left[S P^{\prime}\right]=S^{\prime}$,

$$
\begin{aligned}
\operatorname{Str}\left[\operatorname{Con}_{\rho}\left(S P^{\prime}\right)\right] & =_{\mathrm{df}} S \\
\mathbf{A x}\left[\operatorname{Con}_{\rho}\left(S P^{\prime}\right)\right](s) & ={ }_{\mathrm{df}} \quad \exists s^{\prime}: S^{\prime} . \mathbf{A x}\left[S P^{\prime}\right]\left(s^{\prime}\right) \& \rho\left(s^{\prime}\right)=s s
\end{aligned}
$$

2. $\mathrm{Sel}_{\rho}$, the selector determined by $\rho$, is a specification operation of type $\operatorname{Spec}(S) \rightarrow \operatorname{Spec}\left(S^{\prime}\right)$ defined as: for any specification $S P$ with $\operatorname{Str}[S P]=S$,

$$
\begin{aligned}
\operatorname{Str}\left[\operatorname{Sel}_{\rho}(S P)\right] & =S^{\prime} \\
\left.\operatorname{Ax}_{\mathrm{Sel}}(S P)\right]\left(s^{\prime}\right) & { }_{\mathrm{d} f} \operatorname{Ax}[S P]\left(\rho\left(s^{\prime}\right)\right)
\end{aligned}
$$

Intuitively, the constructor Con $_{\rho}$ applied to specification $S P^{\prime}$ constructs as its realizations the images of $\rho$ over the $S P^{\prime}$-realizations, while the selector Sel applied to $S P$ selects the inverse images of the $S P$. realizations by $\rho$. Interesting specification operations can be defined by using selectors and constructors. For example, Join and Extend discussed above can be defined in the following way:

- The operation Join $_{s}$ can be defined as

$$
\operatorname{Join}_{S}\left(S P, S P^{\prime}\right)={ }_{\mathrm{d} E} \operatorname{Sel}_{d}\left(S P \otimes S P^{\prime}\right)
$$

where $d=\lambda s: S .(s, s): S \rightarrow S \times S$ is the diagonal function over $S$.

- The operation Extend can be defined as

$$
\operatorname{Extend}\left(S P, E x t_{-} S t r, E x t_{-} A x\right)={ }_{\mathrm{df}} \operatorname{Join}_{S}\left(\operatorname{Sel}_{\pi_{\mathrm{I}}}(S P),\left(S, E x t_{-} A x\right)\right)
$$

where $S=\Sigma s: \operatorname{Str}[S P] . \operatorname{Ext} S \operatorname{Str}(s)$ and $\pi_{1}: S \rightarrow \operatorname{Str}[S P]$ is the first projection function.

Another example is that constructors can be used to play a role of 'renaming' and information hiding similar to the operation derive in specification language ASL [SW83, Wir86]. derive in ASL is based on a signature morphism from the signature of the resulting specification to that of the argument specification. Such an signature morphism $\sigma$, when it is a signature inclusion, corresponds to a (forgetful) map $\rho$ from the structure type of the argument specification to that of the resulting specification; and in such a case, Con ${ }_{\rho}(S P)$ corresponds to derive $S P$ from $\sigma$. Similarly, the operation translate (see [ST88a]) can be simulated as selectors.

It is also easy to verify the following basic properties of constructors and selectors.

\footnotetext{
${ }^{8}$ The name 'constructor' comes from the similarity of this class of specification operations with Sannella and Tarlecki's notion of constructor. See section 4.3 .
} 
Proposition 4.6 Let $\rho: S^{\prime} \rightarrow S$.

- Realizability:

1. if $S P^{\prime}: \operatorname{Spec}\left(S^{\prime}\right)$ is realizable, so is $\operatorname{Con}_{\rho}\left(S P^{\prime}\right)$;

2. if $\operatorname{Sel}_{\rho}(S P)$ is realizable, so is $S P$.

- Monotonicity:

1. for $S P_{1}^{\prime}, S P_{2}^{\prime}: \operatorname{Spec}\left(S^{\prime}\right), S P_{1}^{\prime} \Longrightarrow S P_{2}^{\prime}$ implies $\operatorname{Con}_{\rho}\left(S P_{1}^{\prime}\right) \Longrightarrow \operatorname{Con}_{\rho}\left(S P_{2}^{\prime}\right)$;

2. for $S P_{1}, S P_{2}: \operatorname{Spec}(S), S P_{1} \Longrightarrow S P_{2}$ implies $\operatorname{Sel}_{\rho}\left(S P_{1}\right) \Longrightarrow \operatorname{Sel}_{\rho}\left(S P_{2}\right)$.

\subsection{Constructor/selector implementation}

The constructor operations are very similar in spirit to the notion of constructors (functions between algebra classes) introduced in [ST88b], although they are semantically different. Sannella and Tarlecki have proposed the idea to suggest the following refinement methodology: starting from an initial specification $S P$ to be implemented, one uses constructors to specify some specification which implements $S P$ and goes on to implement the argument specifications of the constructors used in this step. Such a method applies to our setting as well. In fact, we can define a similar notion of constructor/selector implementation, which turns out to be equivalent to the notion of implementation we have defined. This enables us to relate our approach to that in algebraic specifications and gives a better understanding of the notion of implementation.

Definition 4.7 (constructor/selector implementation) Let $S P$ and $S P^{\prime}$ be specifications and $\rho$ be of type $\operatorname{Str}\left[S P^{\prime}\right] \rightarrow \operatorname{Str}[S P]$.

1. $S P$ is implemented by $S P^{\prime}$ through constructor $\rho$ (notation $\left.S P \stackrel{c}{\Longrightarrow}_{p} S P^{\prime}\right)$ if $S P \Longrightarrow \mathrm{Con}_{p}\left(S P^{\prime}\right)$.

2. $S P$ is implemented by $S P^{\prime}$ through selector $\rho$ (notation $S P \Rightarrow_{\rho} S P^{\prime}$ ) if $\operatorname{Sel}_{\rho}(S P) \Rightarrow S P^{\prime}$.

Proposition 4.8 Let $S P$ and $S P^{\prime}$ be specifications and $\rho: \operatorname{Str}\left[S P^{\prime}\right] \rightarrow \operatorname{Str}[S P]$. Then, the following are equivalent:

- $S P \Longrightarrow_{\rho} S P^{\prime}$.

- $S P \stackrel{c}{\Longrightarrow} S P^{\prime}$.

- $S P \stackrel{\Leftrightarrow}{\Leftrightarrow} S P^{\prime}$.

Proof The first and the last statements are intensionally equal (convertible) and they are logically equivalent to the second.

Constructors and selectors are essentially dual operations. They suggest different methodologies of refinement development of programs. The notion of selector implementation suggests a bottom-up approach to refinement, while that of constructor implementation suggests a top-down approach, which is usually taken. 


\section{Parameterized Specification}

Parameterization is a powerful abstraction tool both for modular design and for structured specification. A type theory with good structural facilities can provide powerful higher-order parameterization mechanisms for parameterized specifications as well as parameterized program modules.

In fact, we have seen an example in section 4.1 , where we considered implementation of a specification of the form $\Phi\left(S P_{0}, P\right)$. We pointed out there that there are at least two design decisions such a specification form may suggest: one is to decompose it into two independent specifications $S P_{0}$ and $S P^{\prime}$, where $S P^{\prime}$ is a specification of parameterized program modules; another is to directly use the monotonicity property of the specification operation $\sum$ to consider further refinements of the specification $S P_{0}$ and the parameterized specification $P$. Taking this latter view, we must consider parameterized specifications and their implementations.

Of course, the need and usefulness of parameterized specifications in modular design and structuring specifications can not only be explained by a simple example. We would not elaborate this in this paper. Among the large amount of literature on this are [BG80, SW83, EM85] and in particular [SST90] where a recent nice account on this issue can be found.

\subsection{Parameterized specifications}

Parameterized specifications are functions in the type theory which applied to its arguments return specifcations as results. In other words, parameterized specifications have types of the following forms:

$$
\Pi x_{1}: A_{1} \ldots \Pi x_{n}: A_{n} . \text { SPEC } \text { or } \Pi x_{1}: A_{1} \ldots \Pi x_{n}: A_{n} . \operatorname{Spec}(S)
$$

where $n \geq 1$. Note that the forms of arguments to which a parameterized specification can apply are not restricted here; they can be any kinds of objects including structures (program modules), specifications and any knids of parameterized objects.

For example, we may parameterize the specification of stacks (see Example 3.3) in two different ways. First, given any (non-empty) concrete data type $A$ with a congruence relation, the parameterized specification returns a specification of stacks for that concrete data type. This can be done in the obvious way in our type theoretic setting, for example, the stack parameterized over concrete data types would look like

$$
\text { Stack }=\lambda X: T y p e \lambda x: X \lambda R: X \rightarrow X \rightarrow \text { Prop. Stack }(X, x, R)
$$

where $\operatorname{Stack}(X, x, R)$ is the same as $\operatorname{Stack}(\mathrm{N})$ except that $\mathrm{N}, 0$ and $=_{\mathrm{N}}$ are replaced by $X, x$ and $R$, respectively. (Depending on different intentions of how Stack is to be used, one may require that $R$ be a congruence by adding another argument to Stack or remove the argument $R$ by using the Leibriz's equality over $X$.) Such a parameterization is over concrete program modules and the parameterized specification $P$ involved in specification of the form $\Sigma\left(S P_{0}, P\right)$ is of such a kind.

Considering structured specifications and modular design, we may parameterize a specification over specifications. This is what it normally means by parameterized specification in the algebraic approach to 
specifications (c.f., Clear [BG80] and other specification languages). For example, given any specification of an abstract data type (e.g., of sets, stacks or arrays), we may want to extend thera by a specification of stacks to get a specification of stacks of sets, stacks or arrays, etc.. Instead of doing them one by one, we want to parameterize the specification of stacks over such specifications. The example below explains how this can be done.

There is something to say before we give the example. A parameterized specification STACK extending specifications by stacks can not take an arbitrary specification as its argument; the structure type of an eligible argument specification must have a distinguished type with some object. In the algebraic approach to specifications, this is usually done by considering a special specification (usually called Elem) as the parameter specification, which has only one sort and one constant of the sort (see [BG80] for example). Satisfaction (or matching) of an argument specification to a parameter specification is through a signature morphism from the parameter specification to the argument. ${ }^{9}$ In other words, we need to talk about the 'components' of the structure type of specifications. A way to do this is in the type theory is to use functions to indicate the components of a structure type. For example, for any type $S$, a function of type

$$
\operatorname{Elem}(S)={ }_{\mathrm{df}} S \rightarrow \sum[X: \text { Setoid, } x: \operatorname{Dom}[X]]
$$

can be used as a component indicator which, given any $S P$-structure, identifies a type (with a binary relation) and an object of the type. For instance, the following function (c.f., Example 3.6)

$$
i n d \_a r r a y=\lambda A: \operatorname{Str}[\operatorname{Array}(\mathbf{N})] \cdot(\operatorname{Array}[A], \text { newarray }[A])
$$

is of type Elem(Array(N)) and may be used in application of parameterized specification STACK below to generate a specification of stacks of arrays of natural numbers.

Example 5.1 (STACK) We define a parameterized specification STACK which, when applied to a specification whose structure type has a distinguished non-empty setoid, returns as result a specification which extends the the argument specification by a stack specification over the indicated setoid. We shall use the specification operation Extend to define STACK. First, we define two preliminary functions for extensions of structure type and axioms, respectively.

- Ext_Str_Stack is a function of type $\Pi S: T y p e . \operatorname{Elem}(S) \rightarrow S \rightarrow$ Type.

Given a type $S$, a function Elem of type $\operatorname{Elem}(S)$ and an object $s$ of type $S, E x t \_S t r \_S t a c k(S, E l e m, s)$ is defined to be the same as the structure type of Stack(N) in Example 3.3 except that we replace $N$ and $=_{N}$ by $\operatorname{Dom}[X[E l e m(s)]]$ and $E_{q}[X[E l e m(s)]$, respectively.

- Ext_Str_Stack is function of type ПS:Typeח Elem:Elem(S). Es:S.Ext_Str_Stack $(S$, Elem, s) $\rightarrow$ Prop.

\footnotetext{
${ }^{9}$ Note that there is an important difference between the algebraic approach and a type-theoretic approach. In the former, parameter specifications, which play a role of 'type', are at the same level with the actual specification arguments. In a type theory, this can not be the case.
} 
Given a type $S$, Elem of type $\operatorname{Elem}(S)$ and an object $s^{\prime}$ of type $\left.\Sigma s: S . E x t \_S t r \_S t a c k(S, E l e m, s)\right)$, Ext_Str_Stack $\left(S, E l e m, s^{\prime}\right)$ is the proposition defined the same as the axiom part of Stack $(\mathrm{N})$ in Example 3.3 except that we replace $\mathrm{N}, 0$ and $=_{\mathrm{N}}$ by $\operatorname{Dom}\left[X\left[\operatorname{Elem}\left(\pi_{1}\left(s^{\prime}\right)\right)\right], x\left[\operatorname{Elem}\left(\pi_{1}\left(s^{\prime}\right)\right)\right]\right.$ and $\operatorname{Eq}[X[\operatorname{Elem}(s)]]$, respectively.

Now, we define parameterized specification STACK as follows:

$$
\begin{aligned}
\text { STACK }= & \lambda S: T y p e \lambda \text { Elem:Elem }(S) \lambda S P: \operatorname{Spec}(S) . \\
& \text { Extend }(S P, \text { Ext_Str_Stack }(S, \text { Elem }), \text { Ext_Ax_Stack }(S, \text { Elem }))
\end{aligned}
$$

which is of type

$$
\Pi S: T y p e \Pi \text { Elem:Elem }(S) . \operatorname{Spec}(S) \rightarrow \operatorname{Spec}\left(\Sigma s: S . E x t \_S t r \_S t a c k(S, E l e m, s)\right)
$$

Applying STACK to, for example, the specification Array(N) (see Example 3.6) with the component indicator ind_array defined above will result in $\operatorname{STACK}(\operatorname{Str}[\operatorname{Array}(\mathbf{N})]$, ind_array, $\operatorname{Array}(\mathbf{N}))$, the specification of stacks of arrays of natural numbers.

\subsection{Implementation of parameterized specifications}

In a design process, it is often natural to decompose a specification into several specifications some of which are parameterized specifications. For example, when a specification is of the form $P(S P)$ or $\Sigma(S P, P)$, a decomposition into $S P$ and parameterized specification $P$ may be desirable. Such a need calls for a notion of implementation between parameterized specifications.

Definition 5.2 (implementation of parameterized specifications) Let $P$ and $P^{\prime}$ be parameterized specfications over the same parameter type $P$ ar. A refinement map from $P^{\prime}$ to $P$ is a function

$$
\delta: \Pi s: P a r . \operatorname{Str}\left[P^{\prime}(s)\right] \rightarrow \operatorname{Str}[P(s)]
$$

such that the following satisfaction condition is provable:

$$
\operatorname{Sat}(\delta)=_{\mathrm{df}} \forall s: P a r . P(s) \Longrightarrow_{\delta(s)} P^{\prime}(s)
$$

If $\delta$ is a refinement map from $P^{\prime}$ to $P$, we say that $P$ refines to (or is implemented by) $P^{\prime}$ through $\delta$, notation $P \Longrightarrow_{\delta} P^{\prime}$.

Remark A parameterized specification $P$ is implemented by $P^{\prime}$ if $P^{\prime}$ implements $P$ pointwisely through a uniform refinement map. The essential idea of pointwise implementation comes from [SW83]. Note that the polymorphism and type dependency in type theory gives a nice way to express a (uniform) family of refinement maps as a single polymorphic function. One may have noticed the similarity of this definition with the notion of natural transformation between functors in category theory. Although the above definition is 
already rather general, one may further consider implementation between two parameterized specifications with possibly different parameter types. We do not expand this discussion here.

The above notion of implementation composes vertically.

Proposition 5.3 (vertical composition) Let $P, P^{\prime}$ and $P^{\prime \prime}$ be parameterized specifications with the same parameter type Par. If $P \Longrightarrow_{\delta} P^{\prime}$ and $P^{\prime} \Longrightarrow_{\delta^{\prime}} P^{\prime \prime}$, then $P \Longrightarrow_{\delta \bullet \delta^{\prime}} P^{\prime \prime}$, where $\delta \bullet \delta^{\prime}=\lambda s: P a r . \delta(s) \circ \delta^{\prime}(s)$.

Example 5.4 Following example 5.1, we can similarly define a parameterized specification (c.f., Example 3.6)

$$
\begin{aligned}
& \text { ARRAY }=\text { dr } \lambda S: T y p e \lambda E l e m: \operatorname{Elem}(S) \lambda S P: \operatorname{Spec}(S) . \\
& \text { Extend }\left(S P, E x t \_S t r \_A r r a y(S, E l e m), E x t \_A x \_A r r a y(S, E l e m)\right)
\end{aligned}
$$

ARRAY $(S, E l e m, S P)$ extends the argument specification $S P$ by a array specification. For any type $S$ and any component indicator Elem of type $\operatorname{Elem}(S)$, we can find a refinement map $\delta$ (c.f., Example 3.6) such that $\operatorname{STACK}(S$, Elem $) \Longrightarrow_{\delta} \operatorname{ARRAY}(S$, Elem $)$.

There are two kinds of parameter types which often occur: the structure type of some specification, which we have seen in a specification of the form $\Sigma(S P, P)$, or a type of specifications (e.g., $\operatorname{Spec}(S)$ or $\operatorname{SPEC})$. In the latter case, it is important to consider the property of horizontal composition of the implementation relation [BG80], since it guarantees we can implement a specification of the form $P(S P)$ by implementing $S P$ and the parameterized specification $P$ separately. The above notion of implementation also enjoys the property of horizontal composition, when a parameterized specification is monotone with respect to the implementation relation between specifications.

Definition 5.5 (monotonicity) Let Par be SPEC or $\operatorname{Spec}(S)$. A parameterized specification $P$ of type $P a r \rightarrow$ SPEC is monotone if and only if there is a function

$$
f: \Pi A, B: P a r .(\operatorname{Str}[B] \rightarrow \operatorname{Str}[A]) \rightarrow(\operatorname{Str}[P(B)] \rightarrow \operatorname{Str}[P(A)])
$$

such that $\forall A, B: P a r \forall \rho: \operatorname{Str}[B] \rightarrow \operatorname{Str}[A] .\left(A \Longrightarrow_{\rho} B\right) \supset\left(P(A) \Longrightarrow_{{ }_{(\rho)}} P(B)\right)$ is provable. If so, we say $P$ is monotone via. $f$.

Proposition 5.6 (horizontal composition) Let Par be SPEC or $\operatorname{Spec}(S), S P, S P^{\prime}: P a r$ and $P, P^{\prime}$ : $P a r \rightarrow$ SPEC. Then, $P(S P) \Longrightarrow_{p} P^{\prime}\left(S P^{\prime}\right)$ for some $\rho$, if the following conditions hold:

1. $S P \Longrightarrow{ }_{p 0} S P^{\prime}$,

2. $P \Longrightarrow_{\delta} P^{\prime}$, and

3. $P$ or $P^{\prime}$ is monotone. 
Proof Define

$$
\rho={ }_{\mathrm{dr}} \begin{cases}f\left(S P, S P^{\prime}, \rho_{0}\right) \circ \delta\left(S P^{\prime}\right) & \text { if } P \text { is monotone via. } f \\ \delta(S P) \circ g\left(S P, S P^{\prime}, \rho_{0}\right) & \text { if } P^{\prime} \text { is monotone via. } g\end{cases}
$$

Then, we have $P(S P) \Longrightarrow_{\rho} P^{\prime}\left(S P^{\prime}\right)$.

The property of horizontal composition shows that we can implement a specification of the form $P_{0}\left(S P_{0}\right)$ by independent refinements $P_{0} \Longrightarrow \delta_{\delta_{1}} \ldots \Longrightarrow_{\delta_{n}} P_{n}$ and $S P_{0} \Rightarrow_{\rho_{1}} \ldots \Rightarrow_{\rho_{n}} S P_{n}$. Doing so, we do not need to verify the monotonicity of more than one of the parameterized specifications, since monotone parameterized specifications can only be implemented by monotone ones.

Proposition 5.7 Let $P$ and $P^{\prime}$ be parameterized specifications over $P$ ar. Suppose $P \Rightarrow_{8} P^{\prime}$. Then, if the parameterized specification $P$ is monotone, so is $P^{\prime}$, and vice versa.

Proof Suppose $P$ is monotone via. $f$. Then $P^{\prime}$ is monotone via. the following function:

$$
g=\mathrm{df} \lambda A, B: P a r \lambda \rho: \operatorname{Str}[B] \rightarrow \operatorname{Str}[A] . \delta(A) \circ f(A, B, \rho) \circ \delta(B)
$$

The other direction is similar.

Finally, we remark that the above definition of monotonicity and proposition for horizontal composition can easily be generalized to the case where parameterized specifications have more than one specification as arguments ( $P a r$ is of the form $\operatorname{Par}_{1} \times \ldots \times \operatorname{Par}_{n}$ where $\operatorname{Par}_{i}$ is either SPEC or Spec $\left(S_{i}\right)$ ).

\section{Conclusions}

We have considered a type-theoretic approach to program specification and data refinement in a type theory with a strong logical power and good structural mechanisms. The higher-order facilities in the type theory provide useful mechanisms for modular design and structured specification. A notable advantage is that we have been able to formalize internally the notions like implementation in the type theory, and this enables us to use an implementation of the type theory (Lego) to develop the refinement maps (programs) and the correctness proofs of implementations. Thus, by means of type theory - a more restricted formalism compared with set theory, we have gained a good compromise between the model-theoretic approaches to specification semantics and implementation, which usually causes difficulties in finding suitable proof development supports, and pure axiomatic approaches which could not talk directly about model sets (and may suffer from a complicated notion of implementation, for example).

Discussions in this paper have omitted an important aspect about specification and implementation, that is, observational equivalence. In our setting, we can consider observational specification as well. For example, Sannella and Tarlecki's notion of abstractor implementation (with observational equivalence as a special case, see[ST88b]) can be similarly dealt with here by introducing the following specification operation: 
- Abstractor: for an equivalence relation $R$ over some structure type $S, \operatorname{Abs}[R]: \operatorname{Spec}(S) \rightarrow \operatorname{Spec}(S)$ is defined as follows:

$$
\begin{aligned}
\mathbf{S t r}[\mathbf{A b s}[R](S P)] & =_{\mathrm{df}} \quad S \\
\mathbf{A x}[\mathbf{A b s}[R](S P)](s) & ={ }_{\mathrm{df}} \quad \exists s^{\prime}: S . \mathbf{A x}[S P]\left(s^{\prime}\right) \& R\left(s, s^{\prime}\right)
\end{aligned}
$$

and the notion of abstractor implementation would be given as:

- Abstractor implementation: $S P$ is implemented by $S P^{\prime}$ via $R$ (an equivalence relation over $\operatorname{Str}[S P]$ ) through refinement map $\rho: \operatorname{Str}\left[S P^{\prime}\right] \rightarrow \operatorname{Str}[S P]$, notation $\stackrel{a}{\Rightarrow}_{\rho}^{R}$, if and only if,

$$
\operatorname{Abs}[R](S P) \Longrightarrow_{\rho} S P^{\prime}
$$

Further research is needed to consider this topic in more details.

Among the related work, the work by Taylor, Pollack and the author on abstract reasoning [TL88, Luo89b] and Burstall's idea on deliverables [BM90] are most influential on this work (in particular, the basic notion of implementation between specifications). Sannella, Sokolowski and Tarlecki [SST90] have recently proposed their ideas of higher-order parameterization and are working on a specification formalism [ST90] which is based on ASL and a type system involving semantical inference. The author has appreciated very much their argument on the difference between parameterized specifications and specifications of program modules and this infuences the careful distinction of these two in the final versoin of this paper. Our idea of using explicit congruence relations in specifications of abstract data types seems to be related to some similar ideas which appeared in algebraic specifications (see [WB89] and [MSV83] for example). The relationship of our approach to that in algebraic specifications is not clear yet.

Finally, there is a very interesting relationship between the researches in program development and in proof development systems which are developed for theorem proving. For example, the refinement proof development style in Lego has many common features compared with program development. A full-scale discussion on this is out of the range of this paper.

Acknowledgements Thanks to Rod Burstall for many suggestions, Don Sannella and Ed Kazmierczak for interesting discussions, and the members in the Lego club in Edinburgh for their comments on this work.

\section{References}

[BB85] C. Böhm and A. Beradurcci. Automatic synthesis of typed $\lambda$-programs on term algebras. Theoretical Computer Science, 39, 1985.

[BCM89] R. Backhouse, P. Chisholm, and G. Malcolm. Do-it-youself type theory. Formal Aspects of Computing, 1(1), 1989.

[Bee82] M.J. Beeson. Problematic principles in constructive mathematics. Logic Colloquiun'80, 1982. 
[BG80] R. Burstall and J. Goguen. The semantics of Clear, a specification language. Lecture Notes in Computer Science, 86, 1980.

[BL84] R. Burstall and B. Lampson. Pebble, a kernel language for modules and abstract data types. Lecture Notes in Computer Science, 173, 1984.

[BM90] R. Burstall and J. McKinna. Deliverables: an approach to program development in the calculus of constructions. In the preliminary Proceedings of the 1st Workshop on Logical Frameworks, 1990.

[C $\left.\mathrm{C}^{+} 86\right]$ R.L. Constable et al. Implementing Mathematics with the NuPRL Proof Development System. Pretice-Hall, 1986.

[CH88] Th. Coquand and G. Huet. The calculus of constructions. Information and Computation, 76(2/3), 1988.

[Chu40] A. Church. A formulation of the simple theory of types. J. Symbolic Logic, 5(1), 1940.

[CPM90] Th. Coquand and Ch. Paulin-Mohring. Inductively defined types. Lecture Notes in Computer Science, 417,1990 .

[dB80] N.G. de Bruijn. A survey of the project AUTOMATH. In J. Hindley and J. Seldin, editors, To H. B. Curry: Essays on Combinatory Logic, Lambda Calculus and Formalism. Academic Press, 1980.

[EFH83] H. Ehrig, W. Fey, and H. Hansen. ACT ONE: an algebraic specification language with two levels of semantics. Technical Report 83-03, Technical University of Berlin, Fachbereich Informatik, 1983.

[EM85] H. Ehrig and B. Mahr. Fundamentals of Algebraic Specification I: Equations and Initial Semantics. Springer, 1985.

[FGJM85] K. Futatsugi, J. Goguen, J.-P. Jouannaud, and J. Meseguer. Principles of OBJ2. Proc. POPL $85,1985$.

[GHM76] J.V. Guttag, E. Horowitz, and D.R. Musser. Abstract data types and software validation. Comm. ACM, 21(12), 1976.

[Gir72] J.-Y. Girard. Interprétation fonctionelle et élimination des coupures de l'arithmétique d'ordre supérieur. PhD thesis, Université Paris VII, 1972.

[GTW78] J.A. Goguen, J.W. Thatcher, and E.G. Wagner. Abstract data types as initial algebras and the correctness of data representation. In R. Yeh, editor, Current Trends in Programming Methodology, Vol. 4. Prentice Hall, 1978. 
[Hoa72] C.A.R. Hoare. Proofs of correctness of data representation. Acta Informatica, 1(1), 1972.

[HP89] R. Harper and R. Pollack. Type checking, universe polymorphism, and typical ambiguity in the calculus of constructions. Theoretical Computer Science, 1989. to appear.

[Hue87] G. Huet. A calculus with type:type. unpublished manuscript, 1987.

[Jon86] C.B. Jones. Systematic Software Development using VDM. Prentice-Hall, 1986.

[LPT89] Z. Luo, R. Pollack, and P. Taylor. How to Use LEGO: a preliminary user's manual. LFCS Technical Notes LFCS-TN-27, Dept. of Computer Science, Edinburgh University, 1989.

[Lu089a] Zhaohui Luo. ECC, an extended calculus of constructions. In Proc. of the Fourth Ann. Symp. on Logic in Computer Science, Asilomar, California, U.S.A., June 1989.

[Lu089b] Zhaohui Luo. A higher-order calculus and theory abstraction. To appear in Information and Computation, 1989.

[Luo90a] Zhaohui Luo. An Extended Calculus of Constructions. PhD thesis, University of Edinburgh, 1990. Also as Report CST-65-90/ECS-LFCS-90-118, Department of Computer Science, University of Edinburgh.

[Luo90b] Zhaohui Luo. A problem of adequacy: conservativity of calculus of constructions over higherorder logic. Technical report, LFCS report series ECS-LFCS-90-121, Department of Computer Science, University of Edinburgh, 1990.

[Lu090c] Zhaohui Luo. A unifying theory of dependent types. manuscript., 1990.

[LZ75] B. Liskov and S. Zilles. Specification techniques for data abstraction. IEEE Trans. on Software Engineering, SE-1(1), 1975.

[Mac81] D.D. MacQueen. Structures and parameterization in a typed functional language. Proc. Symp. on Functional Programming and Computer Architecture, 1981.

[ML75] Per Martin-Löf. An intuitionistic theory of types: predicative part. Logic Colloquium'73, 1975.

[ML82] Per Martin-Löf. Constructive mathematics and computer programming. In Logic, Methodology and Philosophy of Science, VI, 1982.

[ML84] Per Martin-Löf. Intuitionistic Type Theory. Bibliopolis, 1984.

[MSV83] T.S.E. Maibaum, M.R. Sadler, and P.A.S. Veloso. Logical implementation, 1983.

[MTH90] R. Milner, M. Tofte, and R. Harper. The Definition of Standard ML. MIT, 1990.

[NP83] B. Nordström and K. Petersson. Types and specifications. Proceedings of IFIP'83, pages 915-920, 1983. 
[NPS90] B. Nordström, K. Petersson, and J. Smith. Programming in Martin-Löf's Type Theory: an introduction. Oxford University Press, 1990.

[Ore90] C.-H. Ore. The Extended Calculus of Constructions (ECC) with inductive types. draft, 1990.

[PM89] Ch. Paulin-Mohring. Extracting $F^{\omega}$ programs from proofs in the calculus of constructions. Proc. POPL 89, 1989.

[Pol89] R. Pollack. The theory of lego. manuscript, 1989.

[Pol90] R. Pollack. Implicit syntax. In the preliminary Proceedings of the 1st Workshop on Logical Frameworks, 1990.

[Rey74] J.C. Reynolds. Towards a theory of type structure. Lecture Notes in Computer Science, 19, 1974.

[SST90] D. Sannella, S. Sokolowski, and A. Tarlecki. Toward formal development of programs from algebraic specifications: Parameterization revisited. draft, 1990.

[ST87] D. Sannella and A. Tarlecki. Extended ML: an institution-independent framework for formal program development. Proc. Workshop on Category Theory and Computer Programming, LNCS 240, pages 364-389, 1987.

[ST88a] D. Sannella and A. Tarlecki. Specifications in arbitrary institutions. Information and Computation, $76,1988$.

[ST88b] D. Sannella and A. Tarlecki. Toward formal development of programs from algebraic specifications: implementation revisited. Acta Informatica, 25, 1988.

[ST90] D. Sannella and A. Tarlecki. A kernel specification formalism with higher-order parameterization. Draft, 1990.

[SW83] D.T. Sannella and M. Wirsing. A kernal language for algebraic specification and implementation. Technical Report CSR-155-83, Dept of Computer Science, University of Edinburgh, 1983.

[TL88] P. Taylor and Z. Luo. Theories, mathematical structures and strong sums. manuscript, December 1988.

[WB89] M. Wirsing and M. Broy. A modular framework for specification and implementation. TAPSOFT'89, LNCS, 351, 1989.

[Wir86] M. Wirsing. Structured algebraic specifications: a kernel language. Theoretical Computer Science, 42:123-249, 1986. 